\title{
Upgrade of the proton Computed Tomography system of the PRIMA project
}

\author{
Valeria Sipala ${ }^{1}$ \\ Università degli Studi di Sassari \\ Istituto Nazionale di Fisica Nucleare - Catania \\ Via Santa Sofia 64, 95123 Catania - Italy \\ E-mail: valeria.sipala@ct.infn.it
}

\section{Nunzio Randazzo}

Istituto Nazionale di Fisica Nucleare - Catania

\section{Domenico Lo Presti, Concetta Stancampiano}

Università degli Studi di Catania

Istituto Nazionale di Fisica Nucleare - Catania

\author{
Mara Bruzzi, Marta Bucciolini, Stefania Pallotta, Monica Scaringella, Cinzia \\ Talamonti, Mauro Tesi \\ Università degli Studi di Firenze \\ Istituto Nazionale di Fisica Nucleare - Firenze
}

Carlo Civinini, Mirko Brianzi

Istituto Nazionale di Fisica Nucleare - Firenze

\section{Giuseppe Antonio Pablo Cirrone, Giacomo Cuttone, Francesco Romano \\ Istituto Nazionale di Fisica Nucleare - Laboratori Nazionali del Sud}

In this paper a first proprietary apparatus to undergo proton computed tomography (pCT), manufactured in the framework of the PRIMA (PRoton IMAging) project (INFN-CSN5), will be described. The system, able to carry out single projections at different rotating angles of the phantom, is characterized by a field of view of about $5 \times 5 \mathrm{~cm}^{2}$ and an acquisition time of the order of $10 \mathrm{~s}\left(10 \mathrm{kHz}, 10^{5}\right.$ events). The complete system has been characterized with $62 \mathrm{MeV}$ protons at Laboratori Nazionali del Sud (LNS-INFN) and first radiographic and tomographic images have been reconstructed: main results will be shown and discussed. The design and manufacture of a novel prototype of $\mathrm{pCT}$ system for pre-clinical applications is now under development. The system is characterized by: (a) a larger active area and (b) an acquisition system able to store data from a whole tomographic image without dead times. All details will be discussed.

10th International Conference on Large Scale Applications and Radiation Hardness of Semiconductor Detectors Firenze, Italy

July 6-8, 2011

\section{Speaker}




\section{Introduction}

Proton Computed Tomography ( $\mathrm{pCT}$ ) is a medical imaging method based on the use of proton beams with kinetic energy of the order of $250 \mathrm{MeV}$, aimed at directly measuring the stopping power distribution of tissues (presently calculated from X-rays attenuation coefficients) to improve the accuracy of treatment planning in hadron therapy. A pCT system should be able to measure tissue electron density with accuracy better than $1 \%$ and with spatial resolution better than $1 \mathrm{~mm}[1]$.

In past years, there have been different approaches towards the development of proton radiography and tomography apparatus [2,3], but there isn't today a pCT system for clinical applications. The main problem to be faced is the Multiple Coulomb Scattering (MCS) of particles when they interact with matter. The approach proposed here is based on the 'single proton tracking' method with Most Likely Path (MLP) reconstruction of the single particle [4].

Thanks to projects funded by the INFN (CSN5) and MIUR_PRIN 2006, preliminary studies have been carried out and a pCT prototype has been developed and tested with a $62 \mathrm{MeV}$ proton beam at LNS-INFN [5-9]. It includes a tracker, made of silicon microstrip detectors, to measure proton trajectory, and a calorimeter, made of four YAG:Ce optically separated crystals, to measure the residual energy.

Building on acquired skills, the team proposes to construct a pCT system with a field of view and data acquisition time that meet clinical demands.

\section{2. pCT prototype}

The picture of the pCT prototype manufactured is shown in Figure 1: four $\mathrm{x}-\mathrm{y}$ planes of the tracker and the calorimeter can be distinguished. The apparatus has been placed in the beam line at LNS where $62 \mathrm{MeV}$ proton beam are used for eye melanoma treatments [10].

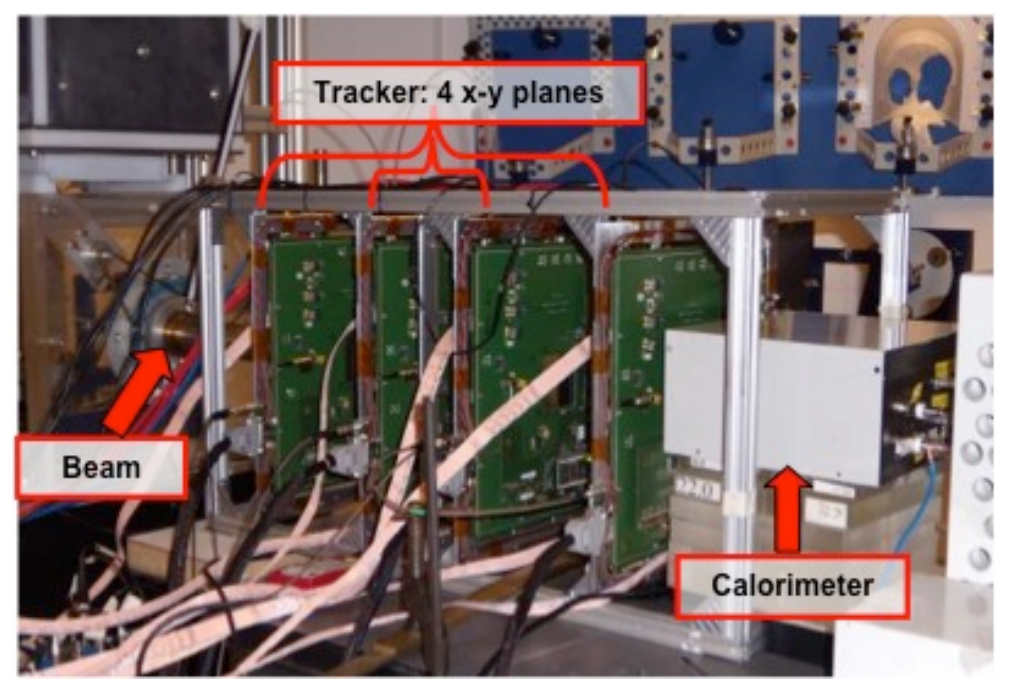

Figure 1 Picture of the pCT prototype in the CATANA ( hadrontherapy center) proton beam line at LNS

The basic idea of the system starts on the relation between the mean energy loss of a proton and the integral of the relative electron density $\rho_{e}$ which can be obtained by integrating the 
reciprocal stopping power $S$, given by the Bethe-Bloch equation, between incident and outgoing energy along the proton's path $L$ :

$$
\int_{E_{\text {in }}}^{E_{\text {out }}} \frac{d E}{S\left(I_{\text {water }}, E\right)}=\int_{L} \rho_{e}(r) d l
$$

The mean excitation potential $\mathrm{I}(\mathrm{r})$, in the stopping power function $S$, was considered constant, equal to the potential of water $\left(I_{\text {water }}=61.77 \mathrm{eV}\right)$. This approximation introduces a relatively small systematic error since most of the traversed object is near-water equivalent soft tissue. Previous equation will hence be the basis of a pCT system because permit to obtain the relative electron density $\rho_{e}$ knowing the stopping power values. For the exact resolution of the integral it is necessary to know the input and output energies, $E_{\text {in }}$ and $E_{\text {out }}$ respectively, and path $L$ of each proton traversing. The path $L$ of the particle inside objects cannot be known exactly because it is affected by Multiple Coulomb Scattering (MCS). In order to derive the best approximation of the particle path an analytical method needs to be developed.

A semi-analytical approach able to predict the mean proton path has been proposed by American researchers active in this field [11]. The best approximation, defined as the Most Likely Path (MLP), is the path which has the same entry point and direction and exit point and direction as the measured trajectory.

These argumentations highlight the fact that for 'single proton tracking' technique application two pieces of information are needed:

- Input and output position and direction for MLP calculus

- Input and output energy of the particle for integral calculus.

So, the pCT apparatus manufactured [5-9] consists of a silicon tracker and a calorimeter with scintillating YAG:Ce crystal array. The tracker includes four x-y planes (P1-P4), each able to measure the $\mathrm{x}-\mathrm{y}$ coordinates at different points of the particle trajectory, while the calorimeter reveals the residual energy of the single proton.

The pCT system manufactured has a field of view of about $5 \times 5 \mathrm{~cm}^{2}$ and acquisition time of the order of $10 \mathrm{~s}\left(10 \mathrm{kHz}, 10^{5}\right.$ events).

Figure 2 shows a drawing of the PMMA phantom placed on beam line, inside the pCT apparatus (between P2 and P3 planes), during the proton test beam at the INFN-LNS (62MeV proton beam). The phantom is $20 \mathrm{~mm}$ thick and it has holes with different diameter and depth as presented in Figure 2.

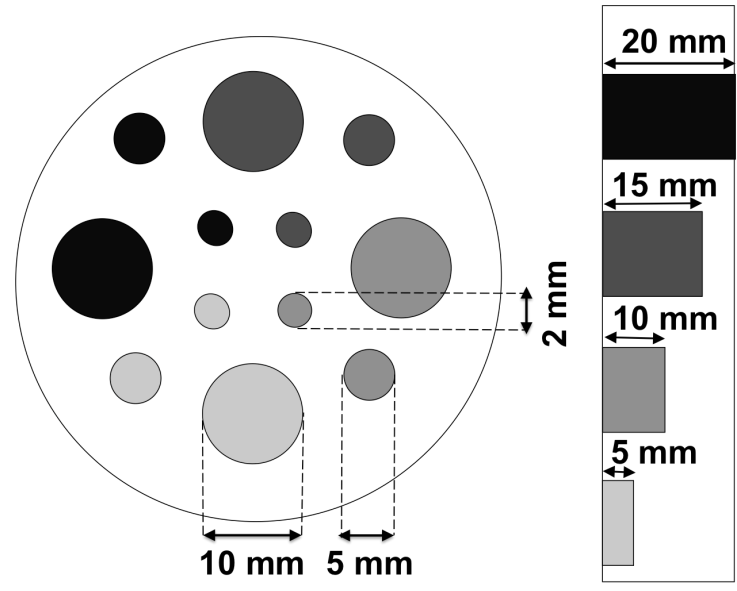

Figure 2 Drawing of the phantom used during proton test beam (62MeV). Same holes with different sizes and depth are manufactured in the area. The holes diameter is comprised between $2 \mathrm{~mm}$ to $10 \mathrm{~mm}$. The minimum hole depth is $5 \mathrm{~mm}$ while its maximum value is $20 \mathrm{~mm}$ (empty hole) 
The phantom image obtained during this test is shown in Figure 3 and it evidences that the system is able to acquire proton radiographies. In the figure the energy values of the particles are plotted using $\mathrm{x}$ and $\mathrm{y}$-coordinates: for empty holes the proton have the maximum value energy.

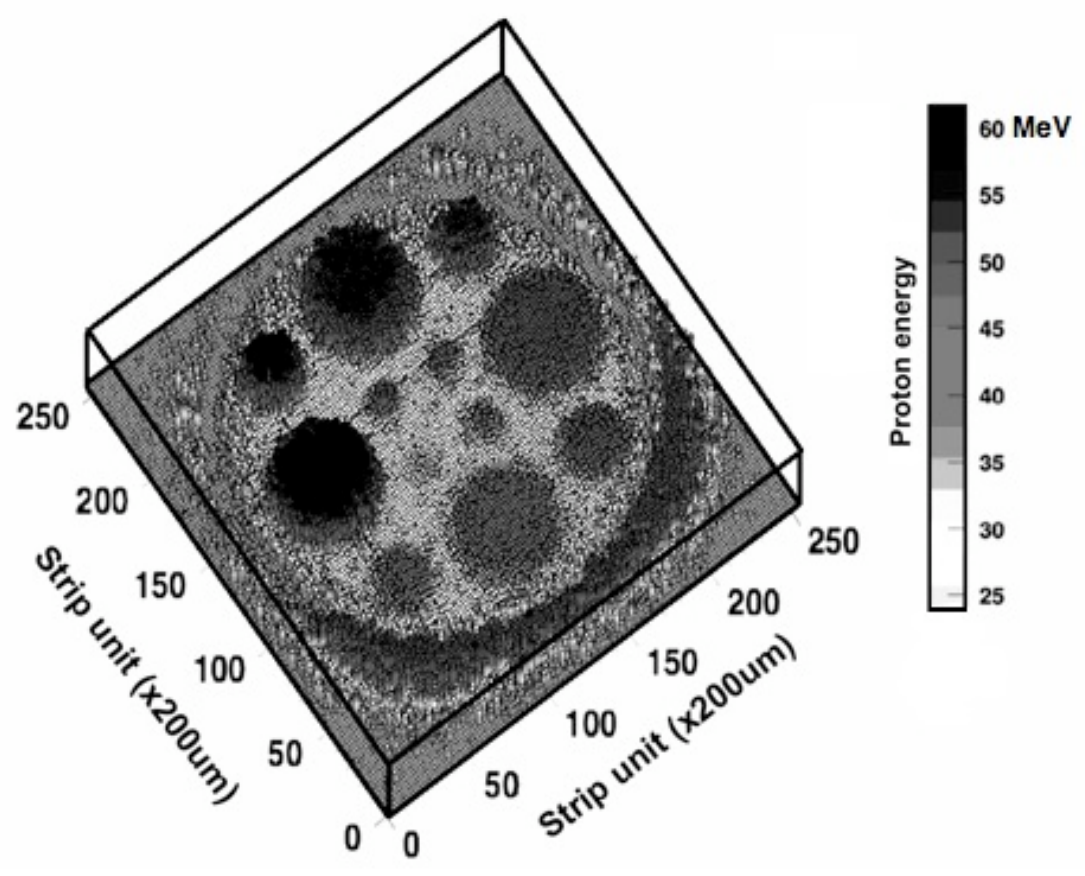

Figure 3 Phantom image obtained using data acquired by the pCT system at LNS (62MeV proton beam) and the MLP reconstruction method

In order to understand the pCT apparatus performances, an image profile is shown in Figure 4. The good spatial resolution is supported by these data: between $55 \mathrm{MeV}$ and $20 \mathrm{MeV}$ the sigma is equal to 3 strips $(600 \mu \mathrm{m})$.

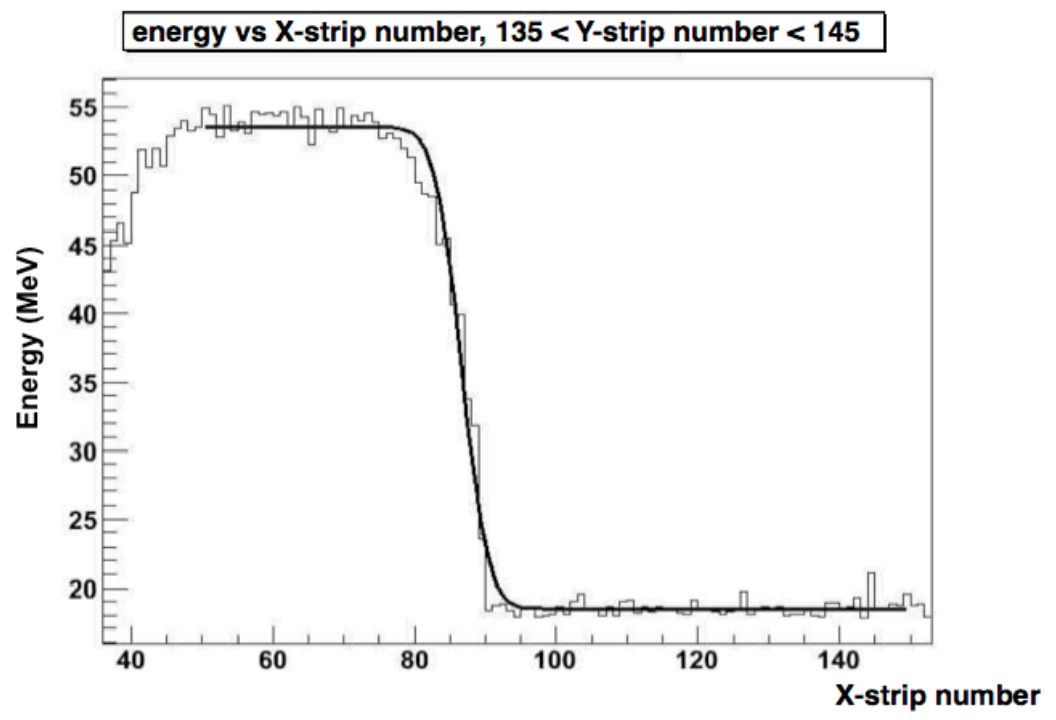

Figure 4 A profile of the phantom image obtained with data acquired by the $p C T$ system and the MLP

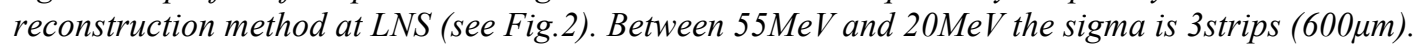


Moreover using a rotating system a tomographic image of a cylindrical phantom has been acquired. The reconstruction algorithm for tomographic image is a crucial point of the pCT development. The PRIMA researchers have performed several studies about the algorithm with MLP reconstruction method [12] and the first tomographic image obtained is shown in [6]. New improvements of algorithm will be possible thanks the data acquired.

\section{Upgrade system}

Building on acquired skills, the team is constructing a pCT apparatus for clinical application based on the same idea but with wider field of view and real-time data acquisition system. New developments are needed as the mechanic system construction, the characterization and assembly of the detectors. The project aims to obtain 3D images with spatial resolution better than $1 \mathrm{~mm}$ and contrast lower than $1 \%$ with $250 \mathrm{MeV}$ proton beam.

The field of view will be extended with respect to the pCT prototype in order to acquire images of phantoms with a size similar to that of a human head.

Also the new pCT system will consist of a tracker and a calorimeter which will be described in the next subsections.

\subsection{Tracker}

In order to simplify the tracker design single-sided sensors have been chosen. Two of these sensors will be coupled together with the strips placed at $90^{\circ}$ to each other leaving a space of 1.5 $\mathrm{mm}$ in a detection plane. The single-sided silicon microstrip sensors are an affordable solution from the point of view of detector cost and reduce the complexity of the entire system thus increasing its reliability.

The pitch of the strips $(200 \mu \mathrm{m})$ has been chosen as compromise between the spatial resolution requirement and the number of read-out electronic channels. The silicon detector thickness introduces a multiple Coulomb scattering contribution to the trajectory measurement which affects the quality of the image cancelling the benefits from having a performing detectors in terms of position resolution. The thickness chosen $(200 \mu \mathrm{m})$ depends on the limits of detector technology and it guarantees that the charge signal of the particle can be detectably to the electronic readout.

The tracker will be made of four identical planes, each able to reconstruct a three dimensional point on the proton trajectory. The technology to directly mount the silicon microstrip sensors on a conventional printed circuit board (PCB) has been validated by the pCT prototype and will be used again. The conceptual design of the plane board is shown in Figure 5 where front and back sides of the circuits are separately shown.

The arrows indicate the position of the micro-bonds and the directions of the strips towards the front-end electronics. The silicon sensors will be glued onto a central hole precisely worked directly onto the PCB material. 

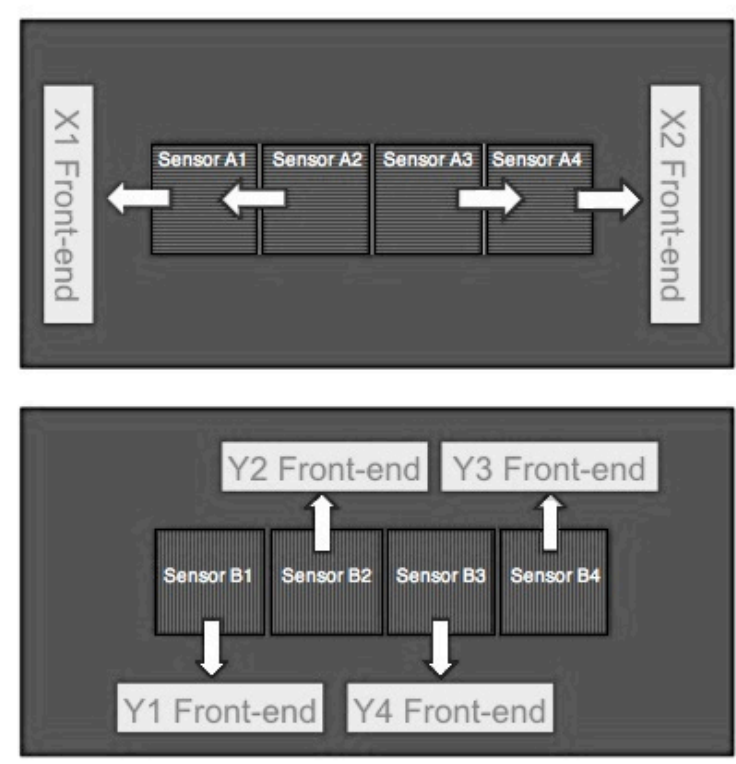

Figure 5 Tracker plane board: conceptual scheme (front and back side of PCB)

Two front-end blocks, with dedicated VLSI front-end chips and FPGAs (Spartan6 XC6SLX45(T) CSG484) for data acquisition, trigger detection and on line zero suppression, will be implemented on the PCB front site (x-plane), while four blocks are needed on the back site (y-plane).

The readout system is illustrated in Figure 6. The detector has to sustain high intensity proton beam (about $1 \mathrm{MHz}$ acquisition rate), so, for VLSI chip a binary multichannel front-end architecture, providing fully parallel signal processing, is the best solution. Each channel will consist of a charge sensitive amplifier, a shaper and a discriminator as the previous chip developed and implemented in the pCT prototype [5-9]. In the new version an internal threshold tuneable by DAC will be implemented in order to circumvent the mismatching problems between different channels, observed during the calibration and the test phases of the pCT prototype. All 256 outputs will be sampled and acquired by the Spartan6 FPGA. All front-end FPGAs will be connected via a high speed serial line to a Central Unit constructed with a commercial development FPGA board (Xilinx ML605). The Central Unit will be connected via the Ethernet link to the DAQ PC for data transfer. The Central Unit will also take charge of the control of the rotation system in order to manage and synchronize all the pCT scanner functions. 


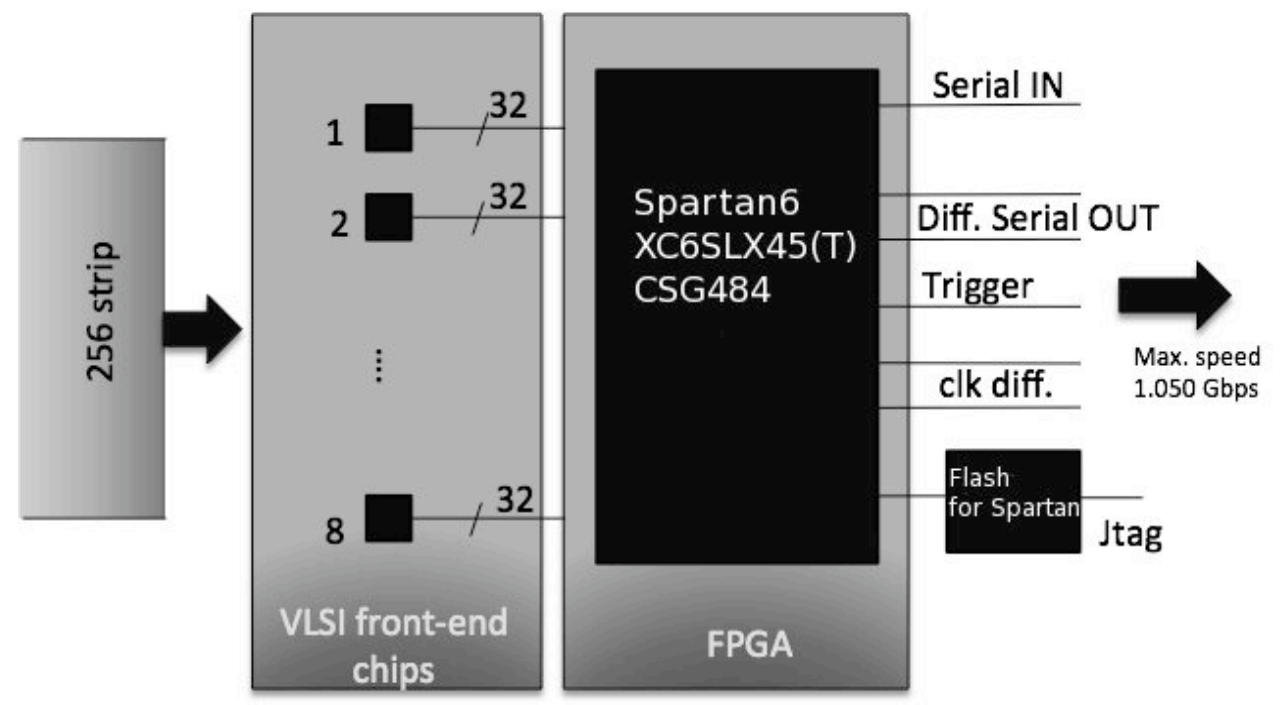

Figure 6 Tracker front-end block conceptual scheme

\subsection{Calorimeter}

The results regarding the calorimeter for residual energy acquisition, obtained with the $\mathrm{pCT}$ prototype, led to a transverse segmented calorimeter being chosen to measure energy. It consists of an array of YAG:Ce scintillator crystals [13] optically separated, each coupled with a photodiode.

The calorimeter is able to sustain a $1 \mathrm{MHz}$ proton rate, because the light decay time of the YAG:Ce crystal is about 70ns. Using photodiodes in the readout system the calorimeter meets the requirement not to be influenced by magnetic fields.

In the pCT system for clinical application the calorimeter must overlap the tracker area $\left(5 \times 20 \mathrm{~cm}^{2}\right)$ to fully cover the entire field of view. The new calorimeter design consists of a $2 \times 7$ array of YAG:Ce crystals, each with a $3 \times 3 \mathrm{~cm}^{2}$ cross-section and $10 \mathrm{~cm}$ long as shown in Figure 7.

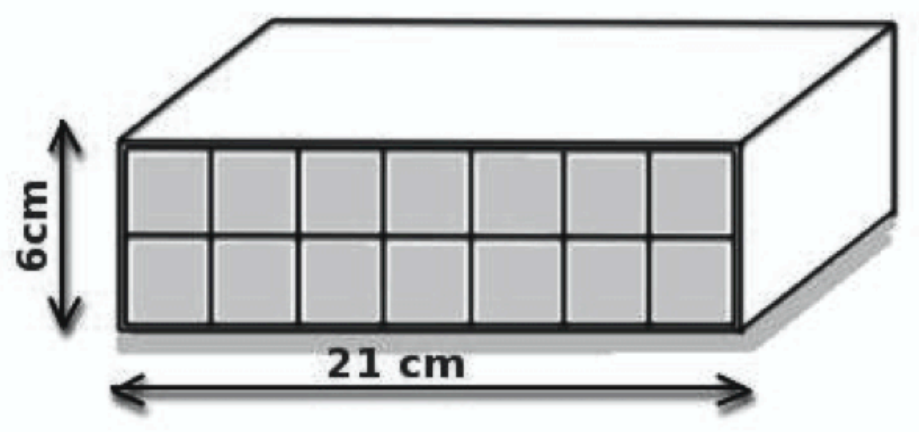

Figure 7 Array of YAG:Ce crystals that will be manufactured as the calorimeter of the pCT apparatus

The first element of the crystal readout channel is the photodiode. The electronic readout channel will consist of a fast charge sensitive amplifier followed by a shaper. 
The trigger of the whole system will be generated by the calorimeter. Each proton that hits one of the crystals will generate a valid event.

The 14 analog outputs from the calorimeter will be sampled by commercial flash ADC with on-board powerful FPGA (National Instruments [14] equipment) that can perform digital real time operations. If one of the signals is over a fixed threshold a trigger and event label (Global Event Number - GEN) will be generated and distributed via a common digital bus. The calorimeter signal amplitude (proportional to the particle energy) and event label will be sent to a DAQ PC via an Ethernet link. In particular, the trigger signal and the GEN will be generated and sent by the 32-Channel Digitizer Adapter Module (NI 5751) to the tracker Central Unit. Moreover the FlexRIO module will provide to pre-elaboration data and, finally, using a $1.9 \mathrm{GHz}$ Intel Celeron T3100 Dual Core With LabVIEW Real-Time (NI PXIe-8102 RT), the calorimeter data will be stored and transferred by Ethernet connection.

\section{Conclusions}

A pCT prototype has been developed and manufactured. The first radiographic and tomographic images have been acquired, evidence of the system functionality. New tests with higher energy proton beam $(200 \mathrm{MeV})$ will be performed by the end of the 2011 .

A new pCT apparatus for preclinical studies has been designed and it is in development phase. The aim is the manufacturing of a pCT system with higher field of view and real-time data acquisition, able to obtain $3 \mathrm{D}$ images with spatial resolution better than $1 \mathrm{~mm}$ and contrast lower than $1 \%$, using $200-250 \mathrm{MeV}$ proton beam.

\section{References}

[1] R.W. Schulte et al. IEEE Trans. Nucl. Sci., Vol. 51, no 3, (2004) 866-872

[2] V.W. Steward and A.M. Koehler, Science (1973) 913 - 914.

[3] A.M. Cormack and A. M. Koehler Phys. Med. Biol. 21 (1976) 560-564.

[4] H.F.W. Sadrozinski, et al., Nucl. Instr. and Meth. A 514 (2003) 215-223.

[5] V. Sipala et al., Nucl. Instr. and Meth. A 658 (2011), 73-77.

[6] G.A.P. Cirrone et al., Nucl. Instr. and Meth. A 658 (2011), 78-83.

[7] V. Sipala et al., Nucl. Instr. and Meth. A, 612 (2010),566-570.

[8] D. Menichelli et al., IEEE Trans. Nucl. Sci., vol. 57, no. 1, (2010) 8-16.

[9] C. Civinini et al., Nucl. Instr. and Meth. A 623 (2010), 588-590.

[10] G.A.P. Cirrone et al., IEEE Trans. Nucl. Sci., vol. 51, no. 3, (2004), 860-866.

[11] D. C. Williams, Phys. Med. Biol., vol. 49, 13 (2004) 2899-2911.

[12] C. Talamonti et al. Nucl. Instr. And Meth. A 612 (2010) 571-575.

[13] V. Sipala et al. Nucl. Instr. And Meth. A 654 (2011) 349-353.

[14] http://italy.ni.com/ 